\title{
A Multiple Channel Access Protocol for Ad Hoc Wireless Networks
}

\author{
Kil-Woong Jang \\ Dept. of Mathematical and Information Science, Korea Maritime University \\ 1 YeongDo-Gu Dongsam-Dong, Busan, Korea \\ Tel: +82-51-410-4375 \\ Fax: +82-51-404-3986 \\ jangkw@bada.hhu.ac.kr
}

\begin{abstract}
We propose a new multiple channel access protocol to enhance the channel utilization and minimize the connection breakage probability in ad hoc wireless networks. In ad hoc networks with multiple channels, communication between a pair of hosts can directly be established using one of available channels. However, hosts' mobility or using channel by neighbors may cause a co-channel interference problem or the connection breakage. To solving these problems, the proposed protocol establishes the new connection using a channel exchange mechanism, which exchanges its available channel with the current channel of the neighboring host without co-channel interference. In addition, it efficiently maintains the current connection of the communicating hosts from co-channel interference caused by hosts' mobility. We evaluate the performance of the proposed protocol using simulation. Simulation results indicate that the proposed protocol may offer performance better than the conventional protocol in terms of the channel utilization and the connection breakage probability.
\end{abstract}

Keywords: ad hoc wireless networks, multiple channel access protocol, co-channel interference

\section{Introduction}

The demand for mobile and direct services has generated interest in ad hoc networks. Ad hoc networks have been designed that mobile host can directly communicate with each other without supporting stationary infrastructures, such as base stations or access points, in conventional wireless networks. In particular, since the stationary infrastructures are hard to establish in situations (i.e., war or natural disasters), mobile users have required the ad hoc service. In conventional wireless networks, if traffic gives overloads to the stationary infrastructures, it may cause high transmission delay and low throughput. On the other hand, mobile hosts, in ad hoc networks, can directly communicate with each other in the transmission range allowed by the transmission power. Due to the transmission range, each host acts as a router, forwarding traffic for other hosts in out of range using routing protocols [6].

Ad hoc hosts have limited channel resources and direct communication between a pair of hosts in the transmission range is established using a same 
common channel. Owing to using limited channel resources, it is important to efficiently utilize channels and reduce connection breakage in ad hoc networks. In IEEE 802.11 protocol for wireless LANs [8], RTS/CTS mechanism is proposed to avoid the occurrence of collision and increase throughput for ad hoc networks. Wireless LANs were typically defined in a single channel system. As the number of communicating hosts increases, systems with a single channel may have the disadvantage which is declination of system performance. However, IEEE Standard 802.11 already has multiple channels available for use. IEEE 802.11b physical layer has 14 channels, $5 \mathrm{MHz}$ apart in frequency. However, to be nonoverlapping and be able to use channels, the frequency must be divided into at most $30 \mathrm{MHz}$. Thus the number of available channels used for communication is 3: channel 1, 6 and 11. On the other hand, IEEE 802.11a has 12 channels, and the number of available channels is 8 for indoor use and 4 for outdoor use, respectively.

There are many related studies for multiple channels [1-5]. Dynamic Channel Assignment (DCA) [2] assigns channels in an on-demand style. This protocol maintains one control channel and other data channels. Each host has two halfduplex transceivers, and one is for control channel and the other is for data channel, respectively. The basic operation in this protocol is similar to IEEE 802.11 protocol. Using RTS/CTS packets, a pair of hosts decides which channel to communicate. Dual Busy Tone Multiple Access [4] has two common channels, which is one control channel and one data channel. To avoid hidden terminals, a control message called busy tone is transmitted on a control channel. In [5], they propose a protocol that is similar to DCA. This protocol also has one control channel and many data channels, and selects the best channel under channel condition of receiver. Anther important issue for multiple channel access protocol is the co-channel interference, which can be occurred due to host mobility or the same channel usage. Previous study [7] was proposed to use a power control mechanism to eliminate the co-channel interference. As the communicating hosts reduce the power of signal, the communication range is reduced and thus the co-channel interference can be eliminated.

In this paper, we propose the multiple channel access protocol to enhance the channel utilization and reduce the connection breakage probability. In the proposed protocol, each host stores the state of channels of communicating neighbors in the communication range and establishes the new communication link using the channel exchange mechanism, which exchanges its available channel with the occupied channel of a communicating neighbor under certain conditions. In addition, the proposed protocol is designed to minimize the co-channel interference caused by host mobility.

\section{Proposed Multiple Channel Access Protocol}

For simplicity, we first describe the basic operation of the proposed protocol using an example of ad hoc networks, as shown in Fig. 1. In this figure, a circle node represents a mobile host. The white-colored host is in idle state and the gray-colored one is in communication state. A dashed line between two hosts 


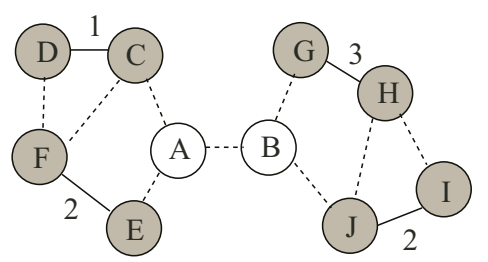

Fig. 1. An example of ad hoc networks.

indicates that each host exists in the communication range and can directly communicate with each other. For example, hosts in the communication range of host A are hosts B, C and E. A line between two gray-colored hosts represents a connecting link between a pair of hosts. A symbol in a circle node represents the address of the host, and the number on the connecting link indicates the channel ID being used by the pair of hosts.

In ad hoc networks with multiple channels, no host can use the channel that is occupied by the communicating neighbors in the communication range owing to the co-channel interference. For example, in Fig. 1, we assume that the total number of available channels in a host is three and then channel IDs are 1, 2 and 3. Hosts $\mathrm{C}$ and $\mathrm{E}$ in the communication range of host $\mathrm{A}$ occupy channel 1 and 2, respectively. Thus, host $\mathrm{A}$ is able to use only channel 3. On the other hand, host B can only use channel 1 . In this situation, the conventional protocol is unable to establish communication between hosts A and B. In other words, a new connection is blocked if no common channel between a pair of hosts exists, and then both must wait until a new common channel is released.

To carry out the proposed protocol, the following operations are positively necessary. After communication is established between a pair of hosts, both inform their neighbors of a connection message. This message includes the communication state of the host, the occupied channel ID and the addresses of communicating hosts. If communication between a pair of hosts is terminated, terminated hosts inform their neighbors of a termination message. Therefore, each host can dynamically specify the state of neighbors from the received messages. To support the operations, each host maintains a table in its memory, which records the channel usage information of the neighbors, to efficiently determine an available channel. This table is called the channel information table. Fig. 2 shows the channel information table of host A in Fig. 1. For example, hosts $\mathrm{C}$ and $\mathrm{D}$ are in a communication state and use channel 1 for communication. Now, we describe the operation of the proposed protocol in the situation of Fig. 1. The proposed protocol is attempted in the following order.

1. Host A broadcasts a connection request message with available channel IDs and the address of host $\mathrm{B}$. On receiving the message, host $\mathrm{B}$ compares the received channel IDs with its channel information. If there is an identical available channel, host $\mathrm{B}$ replies a connection confirm message with the identical channel ID to host A and then executes step 4; otherwise, step 2 is initiated. 


\begin{tabular}{|c|c|c|c|}
\hline Channel ID & Channel state & Address 1 & Address 2 \\
\hline 1 & BUSY & C & D \\
\hline 2 & BUSY & E & F \\
\hline 3 & IDLE & & \\
\hline
\end{tabular}

Fig. 2. Channel information table of host A.

2. Host B sends a check request message with the channel IDs received from host A to its communicating neighbors. The neighbors check whether they can continuously communicate, if they exchange its occupied channel with the received channels. If there are available channels, each neighbor replies a check con firm message with the available channel IDs to host B and then executes step 3; if not, it replies a check confirm message with a NULL value to host $B$ and then executes step 3 .

3. When host $\mathrm{B}$ receives the check confirm message, it replies a connection confirm message, including the available channel ID of host B and the available channel IDs received from neighbors, to host $\mathrm{A}$ and then executes step 4.

4. When host $\mathrm{A}$ receives the connection confirm message, it compares its channel information with the channel IDs received from host B. If there is an identical channel, it selects the channel for communication and sends a connection notification message with the selected channel ID to host B and then executes step 7 ; if not, we perform step 5 .

5. Host A sends a check request message with its available channel IDs to communicating neighbors. The neighbors check whether they are continuously able to communicate, if they exchange its occupied channel with the received channels. If there are available channels, each neighbor replies a check confirm message with the available channel IDs to host A and then executes step 6; otherwise, it replies a check con firm message with a NULL value to host $B$ and then executes step 6.

6. When host A receives the check confirm message, it compares the received channel ID with the channel IDs received from host B. If there is an identical channel, host A selects the channel for communication and sends a connection notification message with the selected channel ID to host B and then executes step 7; if not, we perform step 9.

7. When host $\mathrm{B}$ receives the connection notification message, it checks whether the selected channel is equal to the occupied channel of the neighbors. If so, it sends a change request message with one of available channels in it to the neighbor. The neighbor received the message exchanges its occupied channel with the received channel. After the channel is successfully exchanged, the neighbor replies a change con firm message to host B. Host B received the message sends a channel notification message, including the selected channel ID and addresses of hosts A and B, to the neighbors and host A. 


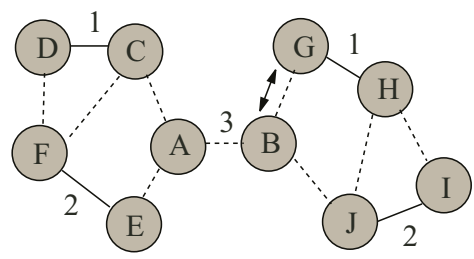

Fig. 3. An example for illustrating the channel assignment after host B exchanges its available channel with the occupied channel of host G.

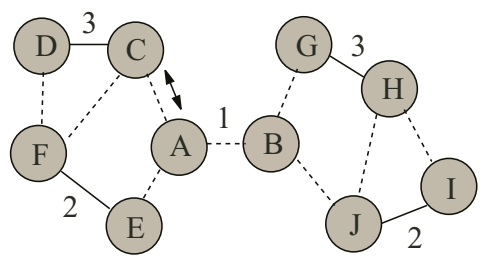

Fig. 4. An example for illustrating the channel assignment after host A exchanges its available channel with the occupied channel of host $\mathrm{C}$.

8. When host A receives the channel notification message, it checks whether the selected channel is equal to the occupied channel of the neighbors. If so, it sends a change request message with one of available channels in it to the neighbor. The neighbor received the message exchanges its occupied channel with the received channel. After the channel is successfully exchanged, the neighbor replies a change confirm message to host A. Host A received the message sends a channel notification message, including the selected channel ID and addresses of hosts $\mathrm{A}$ and $\mathrm{B}$, to the neighbors.

9. If all the above conditions do not hold, then the connection cannot be accomplished. Normally, this would result in the connection being blocked.

As carried out the operation of the proposed protocol, communication between hosts A and B is established as shown in Figs. 3 and 4. During the operation, each host checks if it can exchange its available channels with the occupied channel of neighbors. Simultaneously, it should be checked the state of channels in a host, which is communicating with the neighbor. For example, in Fig. 1, host $\mathrm{G}$ checks if it can exchange the occupied channel of host $\mathrm{H}$ with the channel received from host $B$. If host $\mathrm{H}$ is unable to exchange channels 1 and 3 , host $\mathrm{G}$ is unable to exchange its occupied channel for the channel received from host $\mathrm{B}$. Under this condition, since host B exchanges its available channel with the occupied channel of the neighboring host, Fig. 3 shows that communication between hosts $\mathrm{A}$ and $\mathrm{B}$ can be established. In Fig. 4, since host A exchanges its available channel for the occupied channel of the neighboring host, communication can be established.

In ad hoc networks, each host can directly communicate with each other and move anywhere. As a communicating host moves toward another communicating 


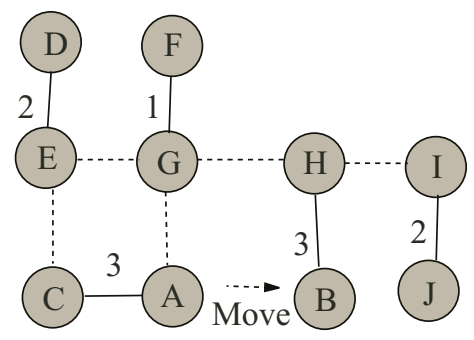

Fig. 5. An example of ad hoc networks for executing the proposed protocol due to host mobility.

host using a same channel, the co-channel interference between both is gradually arisen. For example, in Fig. 5, hosts $\mathrm{A}$ and $\mathrm{C}$ are communicating over channel 3. Hosts $B$ and $H$ are also using the same channel for communication. As host A moves toward host B, the co-channel interference is arisen. In this section, we present the operation of the proposed protocol to minimize the co-channel interference between a pair of hosts due to host mobility.

For simplicity, we describe the protocol operation for host mobility using an example of ad hoc networks, as shown in Fig. 5. The proposed protocol for host mobility is presented below.

1. As host A interferes with host B due to host mobility, hosts A and C check their channel information table and determine a new available channel for channel assignment. If hosts $\mathrm{A}$ and $\mathrm{C}$ have a new available channel, they change their occupied channel to the new channel. Next, hosts A and $\mathrm{C}$ send a channel notification message, including the new channel ID, the addresses of hosts $\mathrm{A}$ and $\mathrm{C}$, to their neighbors, respectively; if not, we perform step 2.

2. Hosts $\mathrm{A}$ and $\mathrm{C}$ send a check request message with their occupied channel ID to their communicating neighbors except host B. The neighbors received the message check whether the co-channel interference occurs, if they exchange their occupied channel for the received channel. If no co-channel interference occurs, each neighbor replies a check confirm message with the received channel ID to hosts A or C; otherwise, it replies a check confirm message with a $N U L L$ value to hosts $\mathrm{A}$ or $\mathrm{C}$.

3. When hosts $\mathrm{A}$ and $\mathrm{C}$ receive the check confirm message, they check if there is an available channel from the received information. If so, they send a change request message with the occupied channel to the neighbor. The neighbor received the message exchanges its occupied channel with the received channel. After the channel is successfully changed, the neighbor replies a change con firm message to hosts $\mathrm{A}$ or $\mathrm{C}$. In addition, hosts $\mathrm{A}$ or $\mathrm{C}$ send a channel notification message, including the new channel ID, addresses of hosts A or C, to their neighbors, respectively; if not, step 4 is initiated.

4. Host A sends a interference request message to host B. On receiving the message, hosts $\mathrm{B}$ and $\mathrm{H}$ check their channel information table and determine a new available channel for channel assignment. If hosts $\mathrm{B}$ and $\mathrm{H}$ have a new 


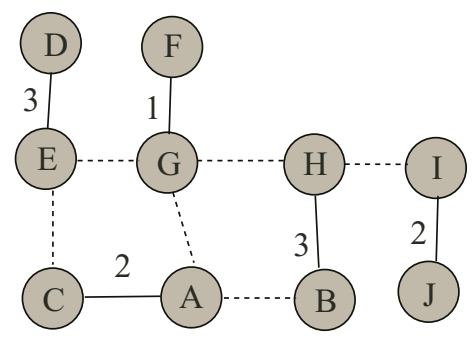

Fig. 6. An example of ad hoc networks after host A changes its occupied channel to a new available channel.

available common channel, they change their occupied channel to the new channel and then host B sends a interference confirm message with the new channel ID to host A. Next, hosts B and $\mathrm{H}$ send a channel notification message, including the new channel ID, the addresses of hosts $\mathrm{B}$ or $\mathrm{H}$, to neighbors, respectively; if not, we perform step 5 .

5. Hosts B and $\mathrm{H}$ send a check request message with their occupied channel ID to their communicating neighbors except host A. The neighboring hosts received the message check whether the co-channel interference occurs, if they exchange its occupied channel with the received channel. If no co-channel interference occurs, each neighbor replies a check confirm message with the received channel ID to hosts $\mathrm{B}$ or $\mathrm{H}$; otherwise, it replies a check confirm message with a $N U L L$ value to hosts $\mathrm{B}$ or $\mathrm{H}$.

6. When hosts $\mathrm{B}$ or $\mathrm{H}$ receive the check confirm message, they check if there is an available channel in the received information. If so, they send a change request message with the occupied channel to the neighbor. The neighbor received the message exchanges its occupied channel with the received channel. After the channel is successfully changed, the neighbor replies a change confirm message to hosts $\mathrm{B}$ or $\mathrm{H}$. Finally, hosts $\mathrm{B}$ and $\mathrm{H}$ send a channel notification message, including the new channel ID, addresses of hosts $\mathrm{B}$ or $\mathrm{H}$, to their neighbors, respectively.

As carried out the operation of the proposed protocol, we assign a new channel to hosts $\mathrm{A}$ and $\mathrm{C}$, as shown in Fig. 6. If all the above conditions do not hold, we are unable to assign a new channel to the interfered hosts. Then, the co-channel interference between hosts A and B occurs.

\section{Performance Evaluation}

In this section we study the impact of the estimation process on the capacity of the proposed protocol through computer simulation. We develop a simulation model to analyze the performance of the proposed protocol under the assumption of ideal channel conditions (i.e., no hidden terminals and capture). The QoS measures that we are interested in are the connection breakage probability, $P_{b}$, and the channel utilization, $U$. 


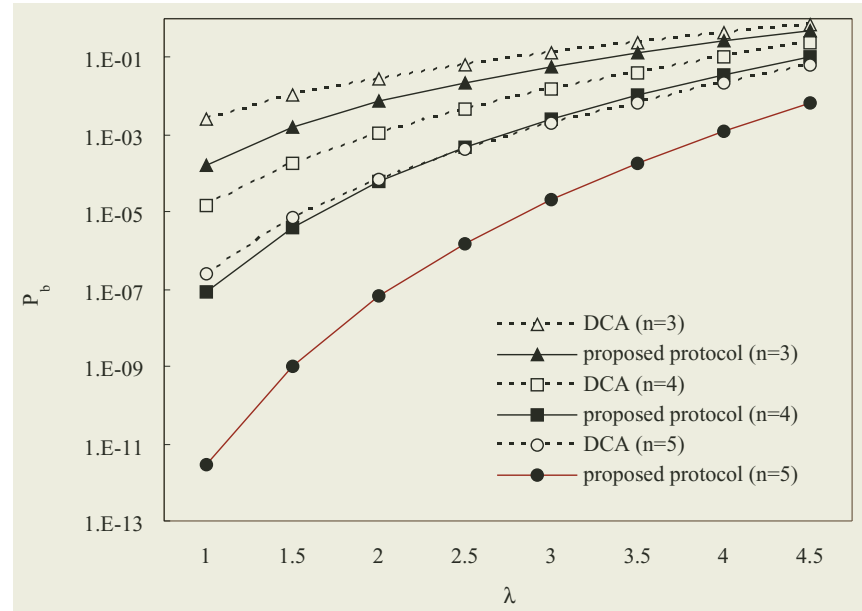

Fig. 7. Connection breakage probability for various values of $n$.

For simulation, we developed a discrete event driven simulator using a high level computer language, $\mathrm{C}++$. The network model for simulation consists of randomly placed 100 nodes in a $1000 \times 1000 \mathrm{~m}$ area. We also consider a mobile network model, where the nodes move independently of one another, which random speeds that are uniformly distributed between $0-20 \mathrm{~m} / \mathrm{s}$. The mobility pattern is based on the random waypoint model [6]. Simulations are performed in wireless LAN environment. The bit rate for each channel is $11 \mathrm{Mbps}$, and the transmission range of each host is approximately $100 \mathrm{~m}$. We assume that each node has a constant bit rate (CBR) traffic and 512 bits control packets, respectively. We also assume that traffic at every node is generated according to Poisson processes with identical mean arrival rates $\lambda$. Each simulation was performed for duration of 60 seconds. The simulation results shown in this paper are valid for up to $95 \%$ confidence intervals.

We provide numerical results, based on simulation, to compare the performance of the proposed and DCA protocols. We obtained values for the connection breakage probability and the channel utilization under different traffic loads $\lambda$, ranging from 1 to 4.5 connections per unit time. Three difference values, 3,4 and 5 , were used for a total number of available channels, $n$, in a host.

Fig. 7 shows $P_{b}$ for the proposed and DCA protocols under varying $n$. In this figure, we can observe that the proposed protocol has better performance than the DCA protocol. When $n$ is increased to 5 , the proposed protocol significantly outperforms the DCA protocol in terms of $P_{b}$. This result can be observed that the $P_{b}$ curve in the proposed protocol is up to four orders of magnitude lower than in the DCA protocol. The reason the proposed protocol provides a lower $P_{b}$ curve is that it can exchange its available channels with the occupied channel of neighbor and thus reduce the connection breakage probability.

Fig. 8 shows the values of $U$ under varying $\lambda$. The proposed protocol achieves uniformly higher values of $U$ under various loads. The difference between the pro- 


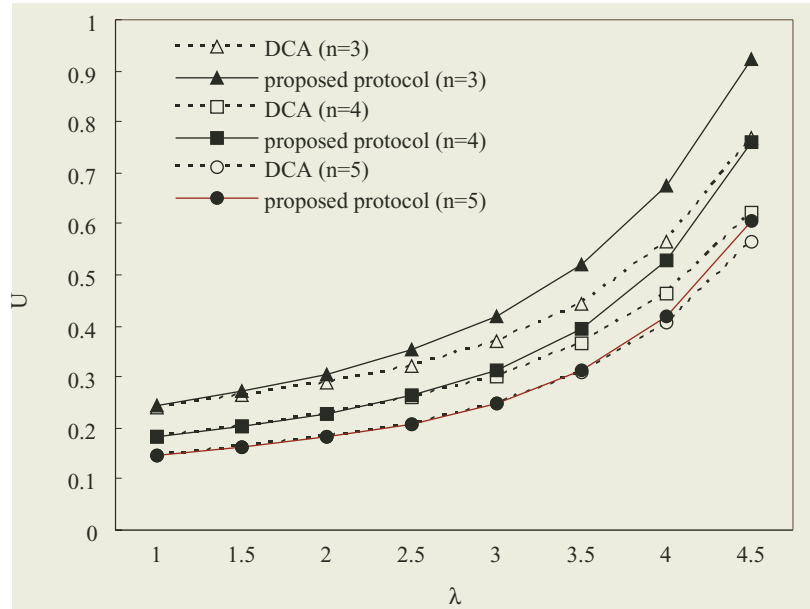

Fig. 8. Channel utilization for various values of $n$.

posed and DCA protocols is most apparent at high loads. Reason the proposed protocol provides a higher $U$ value is that it exploits the channel exchange mechanism. At the same traffic loads, we can see that the protocol in $n=3$ has a higher values than in $n=5$. As $\lambda$ is increased, the probability that hosts use channels in the lower number of available channels is higher than in the higher number of available channels. Therefore, in the lower number of available channels, the $U$ value is higher than in the higher number of available channels. In addition, since the probability of the channel exchange is increased at lower channels, the proposed protocol significantly outperforms the DCA protocol. This result can be observed in Fig. 8, where the proposed protocol at high load is over $10 \%$ more than in the DCA protocol. On the other hand, at $n=5$, the $U$ curve in the proposed protocol is closer than in the DCA protocol. However, the difference in the $U$ curves will be larger as $\lambda$ is increased.

\section{Conclusions}

We have presented a new multiple channel access protocol to improve the system performance in ad hoc wireless networks. In the proposed protocol, each host maintains the channel information and the state of the neighboring hosts. Based on this information, each host carries out a communication with other hosts without the co-channel interference. When the co-channel interference occurs due to host mobility, the proposed protocol minimizes the co-channel interference by allowing hosts to exchange their available channel with the occupied channel of their neighbor. We evaluated the performance of the proposed protocol using simulation. The simulation was focus on the connection breakage probability as well as the channel utilization and then compared with the conventional protocol. The numerical results indicated that the proposed protocol outperformed the conventional protocol over wide range of parameters. 


\section{References}

1. A. Nasipuri, J. Zhuang and S. R. Das, "A multichannel CSMA MAC protocol for multihop wireless networks," Proc. WCNC'99, Sept. (1999) 1402-1406

2. S. L. Wu, C. Y. Lin, Y. C. Tseng and J. P. Sheu, "A new multi-channel MAC protocol with on-demand channel assignment for mobile Ad Hoc networks," Int'l Symposium on Parallel Architectures, Algorithms and Networks, (2000) 232-237

3. C. Y. Chang, P. C. Huang, C. T. Chang and Y. S. Chen, "Dynamic channel assignment and reassignment for exploiting channel reuse opportunities in Ad Hoc wireless networks," IEICE Trans. Commun., vol. E86-B, no. 4, April (2003) 12341246

4. J. Deng and Z. Haas, "Dual Busy Tone Multiple Access (DBTMA): A New Medium Access Control for Packet Radio Network," Florence, Italy, (1998)

5. N. Jain and S. Das, "A Multichannel CSMA MAC Protocol with Receiver-Based Channel Selection for Multihop Wireless Networks," in Proceedings of the 9th Int. Conf. on Computer Communications and Networks (IC3N). Oct. (2001)

6. J. Broch et al., "A Performance Comparison of Multi-Hop Wireless Ad Hoc Network Routing Protocols," in Proceedings of the 4th Int. Conference on Mobile Computing and Networking (ACM Mobicom '98), Oct. (1998) 85-97

7. Y. B. Ko, V. Shankarkumar and N. H. Vaidya, "Medium access control protocols using directional antennas in Ad Hoc network," IEEE INFOCOM 2000, (1999) $13-21$

8. IEEE Standard for Wireless Medium Access Control and Physical Layer Specifications, Aug. (1999) 